\title{
0424 Resistance development in virological failures with DRV/r or LPV/r: 96-week analysis of the Phase III TITAN trial in treatment-experienced patients
}

\author{
S De Meyer*1, E Lathouwers ${ }^{1}$, I Dierynck ${ }^{1}$, E De Paepe $^{1}$, B Van Baelen ${ }^{1}$, \\ T Vangeneugden ${ }^{1}$, F Tomaka ${ }^{2}$, MP de Béthune ${ }^{1}$ and G Picchio ${ }^{2}$
}

Address: ${ }^{1}$ Tibotec BVBA, Mechelen, Belgium and ${ }^{2}$ Tibotec Inc, Yardley, PA, USA

* Corresponding author

from Ninth International Congress on Drug Therapy in HIV Infection

Glasgow, UK. 9-13 November 2008

Published: 10 November 2008

Journal of the International AIDS Society 2008, I I (SuppI I):O46 doi:I0.I I86/I758-2652-II-SI-O46

This abstract is available from: http://www.jiasociety.org/content/I I/SI/O46

(c) 2008 De Meyer et al; licensee BioMed Central Ltd.

\section{Purpose of the study}

In the randomised, controlled, Phase III TITAN trial, at week 96, significantly more patients on darunavir coadministered with low-dose ritonavir $(\mathrm{DRV} / \mathrm{r})$ than on lopinavir/r (LPV/r) achieved HIV-1 RNA $<400$ copies/mL (67.5\% vs. $59.5 \%$; difference $8 \%, 95 \%$ CI $0.1-15.8$ ), confirming non-inferiority $(\mathrm{p}<0.001)$ and superiority of $\mathrm{DRV} / \mathrm{r}$ over $\mathrm{LPV} / \mathrm{r}(\mathrm{p}=0.034)$. A detailed resistance characterisation of virological failures (VFs) was performed.

\section{Methods}

Treatment-experienced, LPV-naïve patients with HIV-1 RNA $>1,000$ copies/mL were randomised to DRV/r 600/ $100 \mathrm{mg}$ BID $(\mathrm{n}=298)$ or LPV/r 400/100 mg BID $(\mathrm{n}=297)$ combined with an optimised background regimen (NRTIs \pm NNRTI). VFs were defined as patients who lost or never achieved HIV-1 RNA < 400 copies/mL after week 16 . Genotyping and phenotyping (Antivirogram ${ }^{\circledast}$ ) were performed by Virco.

\section{Summary of results}

The VF rate in the LPV/r arm $(25.6 \%, \mathrm{n}=76)$ was higher than in the DRV/r arm $(13.8 \%, \mathrm{n}=41)$. Among VFs with an available genotype at baseline and endpoint (72 for $\mathrm{LPV} / \mathrm{r}$ and 39 for DRV/r), more patients developed primary protease inhibitor (PI) mutations at end-point in the LPV/r arm $(n=25)$ than in the DRV/r arm $(n=7)$. Primary PI mutations developing in DRV/r VFs were V32I in three patients, I47V and L76V in two patients and M46I,
I54L, I54M and L90M in one patient. All but the M46I and L90M mutations were 2007 DRV RAMs. In addition, more VFs developed NRTI RAMs in the LPV/r arm $(n=20)$ than in the DRV/r arm $(\mathrm{n}=4)$. Phenotypically, more LPV/r VFs than DRV/r VFs lost susceptibility to the study PI (17/55 vs. $3 / 36)$ or any PI (25/69 vs. $7 / 37)$. Among the DRV/r VFs, the majority retained susceptibility to amprenavir (31/31), atazanavir (29/30), indinavir (31/32), LPV (33/ 33), nelfinavir (24/26), saquinavir (31/31) and tipranavir (34/35). Furthermore, more LPV/r VFs than DRV/r VFs lost susceptibility to the NRTI(s) used in the OBR $(20 / 55$ vs. $4 / 35)$ or any NRTI (27/66 vs. $7 / 38)$. Similar results were obtained when patients with LPV FC $>10$ or patients who previously used $\geq 2$ PIs were excluded from the analysis.

\section{Conclusion}

In this treatment-experienced, LPV-naïve patient population, the overall VF rate with DRV/r was half compared to $\mathrm{LPV} / \mathrm{r}$. Furthermore, the majority of DRV/r VFs did not develop primary PI mutations or NRTI RAMs and preserved susceptibility to PIs and NRTIs. 